\title{
Can Skin Sparing Mastectomy and Immediate Submuscular Implant-Based Reconstruction Be a Better Choice in Treatment of Early-Stage Breast Cancer?
}

(1) Münire Kayahan

Department of General Surgery, University of Health Sciences Turkey, İstanbul Haydarpaşa Numune Training and Research Hospital, İstanbul, Turkey

\begin{abstract}
Objective: To discuss if skin sparing mastectomy (SSM) with immediate submuscular implant-based reconstruction (IBR) can be the preferred treatment in early-stage breast cancer.

Materials and Methods: Patients treated for clinical in situ or early-stage invasive breast cancer with SSM and immediate submuscular IBR between October 2016 and October 2018 were retrospectively evaluated.

Results: Twenty-one cases were reviewed, of whom 18 had two-stage and three had one-stage IBR. Median (range) follow-up period was 42 (32-61) months. Five underwent axillary dissection and 1-2 metastatic nodes were found in three (60\%). Eight patients (38.09\%) with two-stage IBR had radiotherapy because of upstaging and three $(37.5 \%)$ experienced radiotherapy-linked complications. Rate of complications and mean number of events recorded per patient were higher with radiotherapy. Four patients (44\%) had unwanted events after secondary surgery. The mean number of surgeries was higher after two-stage IBR. Mean duration increased in those with chemo-radiotherapy. Six with two-stage and two with one-stage IBR discontinued secondary surgeries.

Conclusion: SSM with immediate submuscular IBR is not suitable in all patients with early-breast cancer. It takes long to have aesthetically pleasing, symmetrical breasts after primary operation because of additional corrective/matching surgeries. Radiotherapy may still be required because of upstaging. Expectation and tolerability of the patient to the process should be evaluated as well as tumor biology and the status of the axilla.

Keywords: breast cancer surgery, immediate breast reconstruction, implant-based reconstruction, direct-to-implant reconstruction, two-stage implantbased reconstructions, breast-conserving therapy
\end{abstract}

Cite this article as: Kayahan M. Can Skin Sparing Mastectomy and Immediate Submuscular Implant-Based Reconstruction Be a Better Choice in Treatment of Early-Stage Breast Cancer? Eur J Breast Health 2022; 18(1): 55-62

\section{Key Points}

- In early-stage invasive breast cancer, mastectomy protects the patient from radiotherapy and its unwanted effects, if upstaging after surgery is not required.

- SSM with immediate submuscular IBR is oncologically safe, but minor and major complications requiring medical and surgical therapies may result. Two-stage IBR is safer but requires at least two operations and several hospital visits for expander inflations.

- To have esthetically pleasing, soft and symmetrical breasts, several ipsilateral and contralateral secondary surgeries are required, which may also cause unwanted events.

- The long duration to reach a satisfying result, extra payments for surgery and devices, extra operations and multiple hospital visits, together with the stress of the main disease can be stressful. Expectations and tolerability of the patient to the process should be evaluated.

\section{Introduction}

In early-stage breast cancer, breast-conserving therapy (BCT), which includes breast-conserving surgery (BCS) and adjuvant radiotherapy, has been preferred to mastectomy as local recurrence rate and overall survival are equivalent. Most patients are pleased to have retained their own breasts but the esthetic outcome is not always satisfying, even after oncoplastic surgery. Fear of recurrence may increase patient stress and exposure of normal tissues to radiation sometimes results in morbidities.

The decision to choose mastectomy has increased in patients with in situ and early-stage breast cancer due to increased use of magnetic resonance imaging (MRI) and genetic testing. Skin sparing mastectomy (SSM) with immediate submuscular implant-based reconstruction (IBR) is an oncologically safe alternative (1). 
In this study, in patients requiring mastectomy because of disease- or patient-characteristics for clinical in situ and early-stage breast cancer, the outcome of those who were treated with SSM and immediate submuscular IBR were retrospectively evaluated. The aim was to assess if SSM with immediate submuscular IBR was superior to BCT in the treatment of early-stage breast cancer by providing psychosocial and esthetic benefits and by negating the need for radiotherapy for a small mass.

\section{Materials and Methods}

Patients operated for clinical in situ and early-stage invasive breast cancer between October 2016 and October 2018 were eligible for inclusion. Inclusion criteria were:

1. Clinical in situ or early-stage invasive breast cancer with preoperative stages of 0 (TisN0), I (T1N0) and IIA (T0N1, T1N1, T2N0)

2. Treatment with SSM, sentinel lymph node biopsy with or without completion axillary lymph node dissection (ALND) and immediate submuscular IBR using a one-stage or two-stage technique.

3. No systemic metastasis, no neoadjuvant therapy.

All patients were re-examined in the breast clinic in May, 2021.

The clinical staging was performed through physical examination, mammography and ultrasonography. Preoperative MRI for the contralateral breast and positron emission tomography scan were performed in all. Tissue diagnoses were performed through core biopsy, fine needle aspiration biopsy or excisional biopsy in cases with a mass and through stereotactic excision in cases with microcalcifications or occult masses.

The choice of mastectomy instead of BCT was made in conjunction with the patient, taking into account lesion characteristics, presence of family or personal history, patient's fear of recurrence and/or in order to avoid radiotherapy. None of the patients had a preoperative genetic test. SSM was performed with removal of the nipple-areolar complex (NAC). Removal of the NAC was decided by the patient to eliminate the risk of recurrence and the need for adjuvant radiotherapy. Nipplesparing mastectomy (NSM) was performed when prophylactic removal of the contralateral breast was performed and there was minimal risk of malignancy or the need for radiotherapy.

Mastectomy was conducted with the pectoral fascia through the subcutaneous adipose tissue. In NSM, the NAC was spared with a thickness of about $2 \mathrm{~cm}$. Sentinel node biopsy was performed through subareolar injection of methylene blue dye. The sentinel nodes were removed through an axillary incision and examined by both intraoperative imprint and postoperative immunohistochemistry. Levels 1-2 completion ALND was added in all cases with any macrometastasis in sentinel nodes and a suction drain was positioned in the axilla. Thoracodorsal vessels were spared if possible.

IBR and all esthetic surgery was performed by the reconstructive surgeon. Two-stage reconstruction was preferred when the surface area was insufficient or when postoperative radiotherapy was expected. A subpectoral pocket was prepared in the avascular plane between the pectoralis major and minor muscles. The lower pole was covered by the elevated serratus anterior muscle or its lower slips. In patients with ptotic breasts, skin reduction was added and inferior 56 dermal-adipose flap was also prepared by deepithelization of the inferior skin. Non-autologous materials to cover the prosthesis were not used in the diseased side, because of the risk of complications delaying adjuvant therapies. The costs of the initial reconstruction and the prosthetic devices were paid for by the Social Insurance Institution (SGK).

Two suction drains were placed, one in the surgical pocket and the other above the muscle, which were removed when the drainage decreased to less than $30 \mathrm{~mL} / 24$ hours. In-patient follow-up occurred in the plastic surgery department for 3-5 days. Antibiotic prophylaxis was started half an hour before the induction of anesthesia and was continued up to the removal of the drains. Supportive brassieres were worn in the operation room and continued through the first two months postoperatively. The tissue expander (TE) was filled with saline once a week after the first fill in the operation room. Inflations were carried on during chemotherapy. Exchange to a permanent implant (PI) was performed after adequate size was achieved by multiple inflations. The PI was postponed until completion of chemotherapy and, when radiotherapy was planned, 4-6 months after completion of radiotherapy.

The necessity and timing of the other esthetic procedures were decided on a per patient-basis by the reconstructive surgeon. Autologous fat grafting was performed under general anesthesia (UGA) to the subcutaneous plane to correct breast contours and deformities. NAC reconstruction included C-V flap for the nipple and tattooing to the nipple/areola. Contralateral matching surgery was performed to correct asymmetry. In contralateral NSM, the PI was placed into the subcutaneous area and covered with biological matrix, which was derived from acellular bovine pericardium.

The requirement for and type of adjuvant therapies were determined by the institutional oncology council. Postmastectomy radiotherapy (PMRT) was applied as intensity-modulated radiotherapy (IMRT) after completion of chemotherapy. Hormone therapy was given when estrogen and/or progesterone receptors were positive. Patients were followed by an oncologist every three months, by the surgeon every six months and, when on hormone therapy, by the gynecologist every six months. The reconstructive surgeon determined appropriate intervals for follow-ups.

This study was approved by the institutional Ethics Committee for Clinical Researches (HNEAH-KAEK 2020/168).

\section{Results}

In total, 21 cases were included in the retrospective analysis. The median (range) age and follow-up period were 48 (37-67) years and 42 (32-61) months, respectively. Six patients $(28.57 \%)$ had a previous history of breast cancer. One had a personal history of contralateral breast cancer. Contralateral mastectomy was added in two cases, one for contralateral widespread microcalcifications and the other for contralateral fibroadenomatosis with ipsilaretal invasive lobular carcinoma (Table 1).

Completion ALND was performed in five (23.8\%) cases. Total numbers of metastatic lymph nodes found were 5/13, 3/15, 2/17, $1 / 11$ and $1 / 16$ (Table 2). Upstaging after surgery was necessary in eight cases $(38.09 \%)$, four in the nodal stage, one in the tumor stage and three for both nodal and tumor staging (Table 3).

All had SSM for the tumor side with submuscular IBR (18 twostage IBR and 3 one-stage IBR). Two women requiring contralateral 
mastectomies underwent SSM in Case 1 and NSM in Case 7 with submuscular two-stage IBR.

Thirteen patients with TE had adjuvant chemotherapy (61.90\%) and eight $(38.09 \%)$ also had adjuvant radiotherapy. Adjuvant chemotherapies were not delayed beyond 1.5 months after the tumor operation (Table 4). Hormone therapy was given to 19 (90.47\%) patients. In the remaining two patients, one was hormone-negative and the other had received anti-estrogen therapy previously. At the time of writing, all 21 patients are alive and disease free.

Mean implant size was $346.66 \mathrm{~mL}$ (between $300 \mathrm{~mL}$ and $390 \mathrm{~mL}$ ) in one-stage and $519.70 \mathrm{~mL}$ (between $375 \mathrm{~mL}$ and $700 \mathrm{~mL}$ ) in twostage cases. Median mean intraoperative fill volume was $141.76 \mathrm{~mL}$ (between $20 \mathrm{~mL}$ and $350 \mathrm{~mL}$ ) and mean number of fills to complete expansion was 7.85 (between 3 and 14). Replacement of the TE with a PI was performed successfully in 14 out of 18 cases. Case 4 is scheduled to have a third PI after removal of the preceding two. Two patients needed removal of the TE because of rupture and one refused the exchange of the inflated TE with a PI.

Complications after primary and secondary surgeries are shown in Table 5.

Seven events were detected in 3/8 (37.5\%) patients who had adjuvant radiotherapy over the subpectoral TE. Case 2 had placement of a new $\mathrm{TE}$ and scoring of the capsule. Case 13 had capsulotomy. In Case 4, exposure of the PI was detected four months after placement and fat grafting. The latissimus dorsi muscle was atrophic and a new PI was placed, which was removed one month later due to wound dehiscence, infection and abscess.

In 6/18 (33.3\%) patients with two-stage reconstruction, complications unrelated to radiation were observed. Dermatitis concurrent with cellulitis was treated with long-term medical therapies. Skin flap ischemia was treated by excision. Ruptured TE was exchanged with a PI in one and removed in two cases. Capsulotomy was performed for capsular contracture. Among the three patients with one-stage reconstruction, two $(66.6 \%)$ had skin flap ischemia and one $(33.3 \%)$ progressed to wound dehiscence. None of the patients had grade IV capsular contracture. Mild to moderate contractures were managed during operations performed for other reasons.

Ipsilateral fat grafting was performed in 12 patients; more than once in three. Dermatitis detected after nipple reconstruction occurred in Case 5 and lasted one month; skin biopsy revealed no malignancy.

Breast-matching surgery was required in 19 cases with unilateral operation. Contralateral NSM and subcutaneous one-stage IBR with acellular matrix was performed 10 months after mastopexy in Case 2, to eliminate the deformities produced by macrocysts, and in Case 16, to relieve the patient's anxiety about contralateral recurrence. Postoperative infection was managed by medical therapy

Table 1. Patient characteristics and co-morbidities with breast signs

\begin{tabular}{|c|c|c|c|c|c|}
\hline Case number & Age of diagnosis (year) & Pre-menopausal & Cancer History & Co-morbidities & Breast sign \\
\hline 1 & 62 & No & Family & DM & $\begin{array}{c}\text { Mass } \\
\text { Calcifications }\end{array}$ \\
\hline 2 & 47 & Yes & - & - & Mass \\
\hline 3 & 65 & No & - & - & Mass \\
\hline 4 & 37 & Yes & - & Smoking & Mass \\
\hline 5 & 46 & Yes & - & - & Mass \\
\hline 6 & 48 & Yes & - & - & Calcifications \\
\hline 7 & 43 & Yes & - & - & $\begin{array}{l}\text { Mass } \\
\text { Mass }\end{array}$ \\
\hline 8 & 56 & No & Personal & - & Calcifications \\
\hline 9 & 51 & Yes & Family & - & Mass \\
\hline 10 & 43 & Yes & - & DM & Mass \\
\hline 11 & 41 & Yes & - & HT & Mass \\
\hline 12 & 46 & Yes & - & Smoking & Mass \\
\hline 13 & 48 & Yes & - & - & Mass \\
\hline 14 & 67 & No & Family & $\mathrm{DM}, \mathrm{HT}, \mathrm{HF}$ & Mass \\
\hline 15 & 54 & No & - & - & Mass \\
\hline 16 & 48 & Yes & - & - & Mass \\
\hline 17 & 52 & No & - & HT & Calcifications \\
\hline 18 & 45 & Yes & - & - & Mass \\
\hline 19 & 45 & No & Family & - & Mass \\
\hline 20 & 45 & Yes & Family & DM & Mass \\
\hline 21 & 48 & Yes & - & - & Mass \\
\hline
\end{tabular}


and debridement on three occasions in one. However, in the other case, removal of the PI was required followed by placement of a TE. Capsulorrhaphy was performed for exposition of the contralateral implant placed during augmentation mammoplasty.

In total 6/18 (33.3\%) who had two-stage reconstruction declined to have the complementary and/or corrective surgery. Among the three patients who refused the second stage, one continued with the expanded TE, and the other two opted for no prosthesis. Two of three (66.6\%) patients with one-stage reconstruction refused all secondary surgery (Table 6).

In patients who completed all the surgery, the mean number of operations UGA and the mean duration are detailed below. One patient with one-stage IBR had four operations UGA within 19 months. The mean (range) number of operations UGA in nine patients with two-stage procedure was 3.5 (2-8). The mean (range) duration in these was 17.5 (11-24) months in two patients who did not receive chemo-radiotherapy, 18 (12-23) months in four patients requiring chemotherapy and 29.3 (24-39) months in three patients requiring chemo-radiotherapy. The mean (range) number of operations performed UGA in the three patients who have not yet completed because of complications is $4.6(3-7)$.

\section{Discussion and Conclusion}

SSM with immediate IBR has a local recurrence rate ranging between $0 \%$ and $8.3 \%$. Recurrence occurs in the subcutaneous tissue at the tumor location in $82 \%$. Survival and local recurrence rates are not worse after NSM, although some glandular tissue is left in situ with the NAC to prevent ischemia (2). The inferolateral pole of the subpectoral implant may be covered with biological matrices or synthetic meshes

Table 2. Clinical and pathological stages with tumor characteristics

\begin{tabular}{|c|c|c|c|c|c|}
\hline Case number & Clinical stage & Pathological stage & Histological type & Tumor subtype & Ki-67 value (\%) \\
\hline \multirow{2}{*}{1} & IL:T1 N0 M0 & IL:T1c N0 Mx & IDC & Luminal A & 28.8 \\
\hline & CL:Tis NO MO & CL:Tis NO Mx & DCIS & ER/PR+ & DCIS \\
\hline 2 & T2 NO MO & $\mathrm{T} 3 \mathrm{mfNO}+\mathrm{Mx}$ & IDC & Luminal A & 14.2 \\
\hline 3 & T2 NO MO & T2mf N1mi Mx & ILC & Luminal A & 40 \\
\hline 4 & T2 NO MO & T2 N1a Mx & IDC & Luminal A & $20-25$ \\
\hline 5 & T1 No MO & T2 N1a Mx & IDC & Luminal A & $30-40$ \\
\hline 6 & T1 No MO & T1a N0 Mx & ILC+ DCIS & Luminal A & $<5$ \\
\hline \multirow{2}{*}{7} & IL:T1 N0 M0 & IL:T1b N0 Mx & ILC+ LCIS & Luminal A & $2-3$ \\
\hline & CL:Benign & CL:Benign & Benign & Benign & Benign \\
\hline 8 & Tis NO MO & Tis NO Mx & DCIS & $\mathrm{ER} / \mathrm{PR}+$ & DCIS \\
\hline 9 & T2 NO MO & T3 N2a Mx & Mixed & Luminal A & $25-30$ \\
\hline 10 & Tis NO MO & Tis NO Mx & DCIS & ER/PR+ & DCIS \\
\hline 11 & T1 N0 M0 & T1c N0 Mx & IDC+DCIS & Luminal A & $20-25$ \\
\hline 12 & Tis NO MO & Tis NO Mx & DCIS & ER/PR- & DCIS \\
\hline 13 & T1 N0 M0 & T1c N1a Mx & IDC & Luminal A & 10 \\
\hline 14 & T1 No M0 & T1C N0 Mx & IDC & Luminal A & $7-8$ \\
\hline 15 & T2 NO MO & T2mf N1mi Mx & IDC & Luminal A & $30-40$ \\
\hline 16 & T1 N0 M0 & T2mf N1a Mx & IDC & Luminal A & 10 \\
\hline 17 & T1 No Mo & T1a N0 Mx & $I D C+D C I S$ & Luminal A & Unknown \\
\hline 18 & T2 NO MO & T2 NO Mx & IDC+DCIS & Luminal A & 9.4 \\
\hline 19 & T1 No M0 & $\mathrm{T} 1 \mathrm{c} \mathrm{N} 0 \mathrm{i}+\mathrm{Mx}$ & ILC & Luminal A & $10-15$ \\
\hline 20 & T1 No MO & T1b N0 Mx & IDC & Luminal A & $7-8$ \\
\hline 21 & T1 N0 M0 & T1b N0 Mx & IDC & Luminal A & 10 \\
\hline
\end{tabular}

IL: Ipsilateral breast, CL: Contralateral breast, IDC: Invasive ductal carcinoma, ILC: Invasive lobular carcinoma, Mixed: Mixed invasive ductal and invasive lobular carcinoma, DCIS: Ductal carcinoma in situ, LCIS: Lobular carcinima in situ, ER: Estrogen receptor, PR: Progesterone receptor

Table 3. Number of patients according to the preoperative clinical and postoperative pathological stages

Stages

Clinical

Pathological

\section{Stage 0 (n)}

3

3

\section{Stage I (n)}

12

9
Stage IIA (n)

6

2
Stage IIB (n)

Stage IIIA (n) 
(3). In the current case series the NAC was removed, ischemic areas were excised early and autologous tissues were used instead of external materials for coverage of the prosthesis. Consequently, adjuvant therapies were started promptly, despite various complications, and no patient had local or systemic recurrence at a mean follow-up period of 43.38 months.

In early-stage invasive and in situ tumors, 65\% of immediate reconstructions in mastectomies are IBRs (4). Two-stage reconstruction is preferred when postoperative radiotherapy is probable. Onestage reconstruction is performed in thin women with small-tomedium, nonptotic breasts when radiotherapy is not expected (5). We performed two-stage IBR in 18 patients who might upstage and one-stage procedure in three patients who were not expected to have radiotherapy. The mean size of the prosthesis in the two-stage procedure was larger.

In patients with lumpectomy who will receive whole breast irradiation, completion ALND is indicated only when three or more sentinel nodes are metastatic or when there are matted nodes intraoperatively (6). The ongoing SENOMAC trial has been randomizing mastectomy patients to either ALND or no ALND (7). The current approach in patients with mastectomy is completion ALND in the presence of any macrometastasis. Our five patients had completion ALND, and in three (60\%), 1 or 2 metastatic nodes were found. If these patients had undergone BCS, they could have avoided ALND and, if tumor biology was favorable, also avoided axillary irradiation. Postponing the analysis of the sentinel nodes to the postoperative period and giving axillary irradiation instead of ALND is another option in patients with mastectomy.

Mastectomy protects the patient from receiving radiotherapy for a small mass with good prognostic features. Radiotherapy makes the resected breast smaller, darker and tough. Exposure of nearby organs can cause rare, aggressive tumors, such as angiosarcoma and myeloid neoplasms, pneumonitis and pulmonary fibrosis, cardiac failure, brachial plexopathy and lymphedema $(8,9)$. Normal tissues can be protected, to some extent, by intraoperative localization of the tumor bed, giving IMRT and using additional techniques during the procedure (10). In older patients, bypassing radiotherapy or giving partial-breast irradiation is controversial (11).

In the present series $13(61.90 \%)$ patients avoided radiotherapy and its adverse effects by undergoing SSM. Eight (38.09\%) patients upstaged and received radiotherapy. PMRT protects from recurrences but nearby organs are exposed to significant amount of radiation (12).

Table 4. Type of primary cancer surgery, prosthesis used, adjuvant therapies and follow-up period

\begin{tabular}{|c|c|c|c|c|c|}
\hline Case number & Primary surgery & Adjuvant CT & Adjuvant RT & Adjuvant HT & Follow-up (month) \\
\hline & $\mathrm{SSM}+\mathrm{SLNB}+\mathrm{TE}$ & & & & \\
\hline 1 & $\mathrm{SSM}+\mathrm{SLNB}+\mathrm{TE}$ & No & No & + & 61 \\
\hline 2 & $\mathrm{SSM}+\mathrm{SLNB}+\mathrm{TE}$ & ST & + & + & 48 \\
\hline 3 & $S S M+S L N B+T E$ & ST & + & + & 47 \\
\hline 4 & $\mathrm{SSM}+\mathrm{ALND}+\mathrm{TE}$ & ST & + & + & 46 \\
\hline 5 & $\mathrm{SSM}+\mathrm{ALND}+\mathrm{TE}$ & ST & + & + & 57 \\
\hline \multirow[t]{2}{*}{6} & $\mathrm{SSM}+\mathrm{SLNB}+\mathrm{PI}$ & No & No & + & 33 \\
\hline & SSM+ SLNB+TE & & & & \\
\hline 7 & $\mathrm{NSM}+\mathrm{SLNB}+\mathrm{TE}$ & $A$ & No & + & 56 \\
\hline 8 & $S S M+S L N B+T E$ & No & No & No & 57 \\
\hline 9 & $\mathrm{SSM}+\mathrm{ALND}+\mathrm{TE}$ & ST & + & + & 44 \\
\hline 10 & $\mathrm{SSM}+\mathrm{SLNB}+\mathrm{TE}$ & No & No & + & 43 \\
\hline 11 & $\mathrm{SSM}+\mathrm{SLNB}+\mathrm{TE}$ & $A$ & No & + & 42 \\
\hline 12 & $\mathrm{SSM}+\mathrm{SLNB}+\mathrm{TE}$ & No & No & No & 42 \\
\hline 13 & $S S M+A L N D+T E$ & ST & + & + & 42 \\
\hline 14 & $\mathrm{SSM}+\mathrm{SLNB}+\mathrm{TE}$ & No & No & + & 42 \\
\hline 15 & $\mathrm{SSM}+\mathrm{SLNB}+\mathrm{TE}$ & ST & + & + & 42 \\
\hline 16 & $\mathrm{SSM}+\mathrm{ALND}+\mathrm{TE}$ & ST & + & + & 38 \\
\hline 17 & $\mathrm{SSM}+\mathrm{SLNB}+\mathrm{PI}$ & No & No & + & 36 \\
\hline 18 & $\mathrm{SSM}+\mathrm{SLNB}+\mathrm{TE}$ & ST & No & + & 34 \\
\hline 19 & $\mathrm{SSM}+\mathrm{SLNB}+\mathrm{TE}$ & A & No & + & 35 \\
\hline 20 & $\mathrm{SSM}+\mathrm{SLNB}+\mathrm{PI}$ & No & No & + & 34 \\
\hline 21 & SSM+ SLNB+TE & $A$ & No & + & 32 \\
\hline
\end{tabular}

SSM: Skin sparing mastectomy, NSM: Nipple sparing mastectomy, SLNB: Sentinel lymph node biopsy, ALND: Axillary dissection, TE: Tissue expander, PI: Permanent breast implant, CT: Chemotherapy, RT: Radiation therapy, HT: Hormone therapy, ST: Sequential use of anthracycline and taxane containing regimens, A: Antracycline regimen 
A carefully performed axillary ultrasound and a core biopsy giving detailed information of the tumor can prevent upstaging. Preoperative ultrasound and positron emission tomography were available in all patients in the present series. An additional ultrasound by the surgeon as an additional check may be safer. The author now confirms preoperative staging by performing an additional ultrasound herself.

In patients with reconstruction, complications and implant failure are detected more frequently when radiotherapy is necessary. The rate of implant failure is higher when TE placement occurs after radiotherapy (13). Giving radiotherapy over the submuscular TE and then replacing it with a PI, with or without latissimus dorsi flap, will be safe. PMRT was given to eight patients who had submuscular TE. Three $(37.5 \%)$ had unwanted events requiring surgical correction. Four of the seven events were detected in one patient and resulted in implant failure. In this case the latissimus dorsi muscle was atrophic. In our patients with two-stage IBR, both the rate of complications requiring surgical corrections and the mean number of events recorded per patient were higher in the eight patients who had radiotherapy than in 10 patients without radiotherapy ( $37.5 \%$ vs. $27.7 \%$ and 2.3 vs. 1.5 , respectively).
Even if no radiotherapy was administered, SSM with submuscular IBR may result in unwanted events, such as hematoma, seroma, skin flap necrosis, infection ranging from cellulitis to sepsis, wound dehiscence and exposure. Explantation is reported to result from infection in $21 \%$ of cases (14). The long-term events may include rupture and deflation of the prosthesis, exposition with asymmetry, capsular contracture, impaired contour, chronic pain and discomfort (15). Besides radiotherapy, obesity, diabetes, smoking, and steroid administration increase complication risk (3). In patients not requiring radiotherapy, the risk for any complication is $52.4 \%$ in the first year and $76.4 \%$ within 8 years. The reoperation rate is reported to increase from $23.3 \%$ within the first year to $40.6 \%$ within 8 years. Skin-flap necrosis, reoperation and extrusion of the implant were more common after one-stage reconstruction $(14,16)$. In our cases who did not have radiotherapy, events requiring surgical corrections in five two-stage IBR patients were: skin flap ischemia; rupture of the TE; and capsular contracture. In two one-stage IBR patients these events included skin flap ischemia and wound dehiscence. In the present case series skin flap ischemia was more common in one-stage IBR (66.6\% vs. $11.1 \%$, respectively).

Table 5. Unwanted events after primary and secondary surgeries in patients with one-stage reconstruction and in those with two-stage reconstruction with and without radiotherapy, and completion of surgeries

\begin{tabular}{|c|c|c|c|c|c|}
\hline \multicolumn{2}{|c|}{ Status } & \multirow{2}{*}{$\begin{array}{c}\text { Case } \\
\text { number } \\
6\end{array}$} & \multirow{2}{*}{$\begin{array}{l}\text { IL events after primary surgery } \\
\text { Skin flap necrosis/ dehiscence }\end{array}$} & \multirow{2}{*}{$\begin{array}{c}\text { CL events after matching } \\
\text { surgeries } \\
-\end{array}$} & \multirow{2}{*}{$\begin{array}{c}\text { Completion of the } \\
\text { surgeries } \\
\text { Discontinued }\end{array}$} \\
\hline no & One-stage & & & & \\
\hline \multirow[t]{7}{*}{$\mathrm{CT} / \mathrm{RT}$} & & 17 & - & Hematoma & Completed \\
\hline & & 20 & Skin flap necrosis & - & Discontinued \\
\hline & Two-stage & 1 & Cellulitis/dermatitis & - & Completed \\
\hline & & 8 & Skin flap necrosis/TE rupture & - & Discontinued \\
\hline & & 10 & TE rupture/ TE removal & - & Discontinued \\
\hline & & 12 & - & Exposition of PI & Completed \\
\hline & & 14 & - & - & Discontinued \\
\hline \multirow[t]{5}{*}{ CT } & Two-stage & 7 & Capsular contracture (BII/III) & - & Completed \\
\hline & & 11 & - & - & Completed \\
\hline & & 18 & - & - & Discontinued \\
\hline & & 19 & - & - & Completed \\
\hline & & 21 & - & - & Completed \\
\hline \multirow[t]{8}{*}{$C T+R T$} & Two-stage & 2 & TE exposition/capsular contracture (BII/III) & $\begin{array}{l}\text { NAC ischemia, infection, } \\
\text { Dehiscence }\end{array}$ & On going \\
\hline & & 3 & - & - & Discontinued \\
\hline & & 4 & $\begin{array}{c}\text { PI } \\
\text { exposure/infection/ } \\
\text { dehiscence/PI removal }\end{array}$ & - & On going \\
\hline & & 5 & Dermatitis & - & Completed \\
\hline & & 9 & - & - & Completed \\
\hline & & 13 & Capsular contracture (BII/III) & - & Completed \\
\hline & & 15 & TE rupture/TE removal (before RT) & - & Discontinued \\
\hline & & 16 & $\begin{array}{l}\text { Skin flap necrosis } \\
\text { (before RT) }\end{array}$ & $\begin{array}{l}\text { NAC ischemia/PI } \\
\text { exposure, infection, PI } \\
\text { removal }\end{array}$ & On going \\
\hline
\end{tabular}


Complications may occur when no breast tissue is left under the thin skin envelope, when the pectoral fascia is removed, or when using complete muscular coverage without an acellular matrix, in addition to other, patient-linked factors. The necessity of removing the pectoral fascia in tumors distant from the fascia is debatable. Case 1, who developed prolonged infection, had diabetes.

Secondary surgery is required following SSM and immediate submuscular IBR in order to achieve esthetically pleasing, soft and symmetrical breasts. These secondary surgeries might include autologous fat grafting, NAC reconstruction, and breast-matching surgery for ptotic, larger or smaller contralateral breasts (17). We performed ipsilateral fat grafting in 12 patients, NAC reconstruction in 10 and contralateral matching surgery in 11 .

Secondary surgery may also result in unwanted events. Four patients (44\%) had events after contralateral matching surgery, two after reduction and augmentation mammoplasties and two after NSM. Acellular matrix, derived from bovine pericardium, was used for coverage of the subcutaneous PI in those with NSM and both had NAC ischemia and infection, resulting in implant failure in one. Subcutaneous PI is usually covered with acellular dermal matrix $(\mathrm{ADM})$ which relieves the pressure on the skin flaps and provides more natural pseudo-ptosis and inferior pole projection compared to a submuscular pocket (18). It decreases the rate of capsular contracture but causes increased seroma formation, implant failure, partial NAC necrosis and rippling (19).

Unwanted events increase both the number of surgeries requiring general anesthetic and the duration before a satisfying result is achieved for the patient. In patients who completed all surgery, the mean number of surgeries UGA was slightly lower in nine patients with two-stage reconstruction compared to one patient with a one-stage procedure
(3.5 vs. 4.0, respectively). The mean duration for completion of all surgery was greater in two-stage patients who had chemo-radiotherapy compared to those who did not. In the three patients undergoing two-stage procedure but who have not yet completed because of complications, the mean number of surgeries was already 3.83 at a mean duration of 43.33 months post initial operation.

Submuscular two-stage reconstruction is safer in cancer patients, but it requires at least two operations with several outpatient visits for expander inflation. ADM-coverage of the lower pole provides more rapid filling, and prevents displacement. ADM use increases the mean intraoperative fill volume from $130.4 \mathrm{~mL}$ to $412.5 \mathrm{~mL}$ and decreases the number of fills needed from 4.3 to 1.7 (20). We did not use ADM on the diseased side and the mean intraoperative fill volume was 141.7 $\mathrm{mL}$ and the mean number of fills was high at 7.85. Rupture of the TE was observed in $16.6 \%$.

SSM and immediate submuscular IBR negate the necessity of having radiotherapy for a small mass and relieve anxiety about recurrence. However, this technique may result in unnecessary ALND, PMRT because of upstaging, extra hospital visits, and extra surgeries UGA, both for complications and to achieve an acceptable appearance. Too many hospital visits, the discomfort from the implants, as well as the cost of secondary surgery and materials may result in exhaustion of the patient, which in turn can lead to discontinuation of secondary surgery or failure to attend follow-up for breast cancer, which is clearly undesirable. The discontinuation rate in our series was $33.3 \%$ in patients with two-stage procedures and $66.6 \%$ in patients with directto-implant IBR.

Although the number of patients in this series was low, it is evident that SSM and immediate submuscular IBR is not suitable in all patients with early-breast cancer. It is important to choose the right patient

Table 6. Performance of primary and secondary surgery

\begin{tabular}{|c|c|c|c|c|}
\hline Type of surgery & & $\begin{array}{l}\text { Two-stage IBR without } \\
\text { RT }\end{array}$ & $\begin{array}{l}\text { Two-stage IBR } \\
\text { with RT }\end{array}$ & One-stage IBR \\
\hline \multirow[t]{2}{*}{ Primary surgery } & Number of patients & 10 & 8 & 3 \\
\hline & Exchange of TE to PI & 8 & 7 & - \\
\hline \multirow{8}{*}{$\begin{array}{l}\text { Secondary surgery } \\
\text { performed }\end{array}$} & IL fat grafting & 6 & 6 & \\
\hline & IL IMF & 2 & & 1 \\
\hline & IL NAC & 6 & 3 & 1 \\
\hline & CL mastopexy & 2 & 1 & \\
\hline & CL augmentation & 1 & 2 & \\
\hline & CL reduction & 2 & 2 & 1 \\
\hline & CL NSM with subcutaneous PI & & 2 & \\
\hline & CL IMF & 2 & & \\
\hline Other surgery & Rhinoplasty & 1 & 1 & \\
\hline \multirow[t]{4}{*}{ Surgery declined } & Replacement of TE with PI & 2 & 1 & - \\
\hline & NAC & 1 & & \\
\hline & NAC and CL corrective surgery & 1 & & \\
\hline & NAC, IL and CL corrective surgery & & & 2 \\
\hline
\end{tabular}

IBR: Implant-based reconstruction, RT: Radiotherapy, TE: Tissue expander, PI: Permanent implant, IL: Ipsilateral, CL: Contralateral, NAC: Nipple-areolar complex reconstruction, IMF: Inframammary fold repositioning, NSM: Nipple-sparing mastectomy, ADM: Acellular dermal matrix 
for the procedure, not only with ultrasound and core biopsy, but also by evaluating the expectations and tolerability of the patient to the process. Cancer patients are very different from patients undergoing reconstructive surgery. It may be better to provide good appearance and an early return to normal life rather than trying to achieve a perfect reconstruction with multiple surgeries, except for those young and tolerant patients with high cosmetic expectations.

Ethics Committee Approval: This study was approved by the institutional Ethics Committee for Clinical Researches of University of Health Sciences Turkey, Haydarpaşa Numune Training and Research Hospital (HNEAHKAEK 2020/168).

Informed Consent: Retrospective study.

Peer-review: Externally peer-reviewed.

Financial Disclosure: The authors declare that this study received no financial disclosure.

\section{References}

1. Juhl AA, Christensen S, Zachariae R, Damsgaard TE. Unilateral breast reconstruction after mastectomy- patient satisfaction, aesthetic outcome and quality of life. Acta Oncol 2017; 56: 225-231. (PMID:28085525) [CrossRef]

2. Galimberti V, Vicini E, Corso G, Morigi C, Fontana S, Sacchini V, et al. Nipple-sparing and skin-sparing mastectomy: review of aims, oncological safety and contraindications. Breast 2017; 34(Suppl 1): S82-S84. (PMID:28673535) [CrossRef]

3. Hallberg H, Rafnsdottir S, Selvaggi G, Strandell A, Samuelsson O, Stadig I, et al. Benefits and risks with acellular dermal matrix (ADM) and mesh support in immediate breast reconstruction: a systematic review and metaanalysis. J Plast Surg Hand Surg 2018; 52: 130-147. (PMID:29320921) [CrossRef]

4. Kummerow KL, Du L, Penson DF, Shyr Y, Hooks, MA. Nationwide trends in mastectomy for early-stage breast cancer. JAMA Surg 2015; 150: 9-16. (PMID:25408966) [CrossRef]

5. Lennox PA, Bovill ES, Macadam SA. Evidence based medicine: alloplastic breast reconstruction. Plast Reconstr Surg 2017;140: 94e-108e. doi: 10.1097/PRS.0000000000003472. (PMID:28654611) [CrossRef]

6. Giuliano AE, Ballman KV, McCall L, Beitsch PD, Brennan MB, Kelemen PR, et al. Effect of axillary dissection vs no axillary dissection on 10-year overall survival among women with invasive breast cancer and sentinel node metastasis: The ACOSOG Z0011(Alliance) randomized clinical trial. JAMA 2017; 318: 918-926. (PMID:28898379) [CrossRef]

7. de Boniface J, Frisell J, Andersson Y, Bergkvist L, Ahlgren J, Rydén L, et al; SENOMAC Trialists' Group. Survival and axillary recurrence following sentinel node-positive breast cancer without completion axillary lymph node dissection: the randomized controlled SENOMAC trial. BMC Cancer 2017; 17: 379. (PMID:28549453) [CrossRef]

8. Horisawa N, Adachi Y, Sawaki M, Hattori M, Yoshimura A, Gondo N, et al. A case of radiation-associated angiosarcoma after breast cancer. Surg Case Rep 2018; 4: 131. (PMID:30406473) [CrossRef]
9. Zeidan AM, Long JB, Wang R, Hu X, Yu JB, Huntington SF, et al. Risk of myeloid neoplasms after radiotherapyamong older women with localized breast cancer: A population-based study. PLoS One 2017; 12: e0184747. doi: 10.1371/journal.pone.0184747. (PMID:28902882) [CrossRef]

10. Stoleru L, Stoleru S, Gaspar B, Noditi A, Blidaru A. Use of a tumor bed boost in the radiotherapy after oncoplastic breast conserving surgery. Chirurgia(Bucur) 2021; 116(Suppl 2): 110-119. (PMID:33963701) [CrossRef]

11. Kunkler IH, Williams LJ, Jack WJ, Cameron DA, Dixon JM; PRIME II Investigators. Breast-conserving surgery with or without irradiation in women aged 65 years or older with early breast cancer (PRIME II): a randomized controlled trial. Lancet Oncol 2015; 16: 26673. (PMID:25637340) [CrossRef]

12. Khan M, Siddiqui SA, Gupta MK, Seam RK, Gupta M. Normal tissue complications following hypofractionated chest wall radiotherapy in breast cancer patients and their correlation with patient, tumor, and treatment characteristics. Indian J Med Paediatr Oncol 2017; 38: 121127. (PMID:28900318) [CrossRef]

13. Oliver JD, Boczar D, Huayllani MT, Restrepo DJ, Sisti A, Manrique OJ, et al. Postmastectomy radiation therapy (PMRT) before and after 2-stage expander-implant breast reconstruction: A systematic review. Medicina(Kaunas) 2019; 55: 226. (PMID:31146506) [CrossRef]

14. Hvilsom GB, Friis S, Frederiksen K, Steding-Jessen M, Henriksen TF, Lipworth L, et al. The clinical course of immediate breast implant reconstruction after breast cancer. Acta Oncol 2011; 50: 1045-1052. (PMID:21604960) [CrossRef]

15. Chen TA, Momeni A, Lee GK. Clinical outcomes in breast cancer expander-implant reconstructive patients with radiationtherapy. J Plast Reconstr Aesthet Surg 2016; 69: 14-22. (PMID:26453182) [CrossRef]

16. Basta MN, Gerety PA, Serletti JM, Kovach SJ, Fischer JP. A systematic review and head-to-head meta-analysis of outcomes following directto-implant versus conventional two-stage implant reconstruction. Plast Reconstr Surg 2015; 136: 1135-1144. (PMID:26595013) [CrossRef]

17. Simonacci F, BertozziN, Grieco MP, Grignaffini E, Raposio E. Autologous fat transplantation for breast reconstruction: A literature review. Ann Med Surg 2016; 12: 94-100.(PMID:27942383) [CrossRef]

18. Woo J, Seung IH, Hong SE. Funnel usefulness in direct-to-implant breast reconstruction using periareolar incision with prepectoral implant placement and complete coverage with acellular dermal matrix. J Plast Reconstr Aesthet Surg 2020; 73: 2016-2024. (PMID:32921621) [CrossRef]

19. Bernini M, Calabrese C, Cecconi L, Santi C, Gjondedaj U, Roselli J, et al. Subcutaneous direct-to-implant breast reconstruction: surgical, functional, and aesthetic results after long-term follow-up. Plast Reconstr Surg Glob Open 2016; 3: e574. (PMID: 26893999) [CrossRef]

20. Sbitany H, Sandeen SN, Amalfi AN, Davenport MS, Langstein HN. Acellular dermis-assisted prosthetic breast reconstruction versus complete submuscular coverage: a head-to-head comparison of outcomes. Plast Reconstr Surg 2009; 124: 1735-1740. (PMID: 19952627) [CrossRef] 